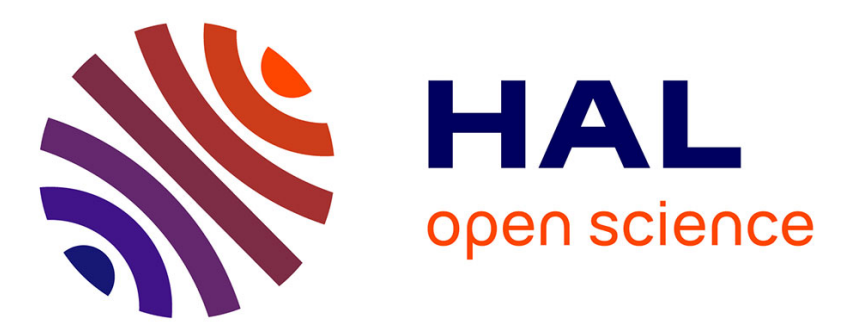

\title{
Conical versus Gaussian terahertz emission from two-color laser-induced air plasma filaments
}

Christian Buhl Sørensen, Christian Buhl Sørensen, Léo Guiramand, Jérôme Degert, Marc Tondusson, Esben Skovsen, Eric Freysz, Emmanuel Abraham

\section{- To cite this version:}

Christian Buhl Sørensen, Christian Buhl Sørensen, Léo Guiramand, Jérôme Degert, Marc Tondusson, et al.. Conical versus Gaussian terahertz emission from two-color laser-induced air plasma filaments. Optics Letters, 2020, 45 (7), pp.2132. 10.1364/OL.390112 . hal-03065559

\section{HAL Id: hal-03065559 https://hal.science/hal-03065559}

Submitted on 14 Dec 2020

HAL is a multi-disciplinary open access archive for the deposit and dissemination of scientific research documents, whether they are published or not. The documents may come from teaching and research institutions in France or abroad, or from public or private research centers.
L'archive ouverte pluridisciplinaire HAL, est destinée au dépôt et à la diffusion de documents scientifiques de niveau recherche, publiés ou non, émanant des établissements d'enseignement et de recherche français ou étrangers, des laboratoires publics ou privés. 


\title{
Conical vs Gaussian terahertz emission from two-color laser-induced air plasma filaments
}

\author{
Christian Buhl Sørensen ${ }^{1}$, Léo Guiramand ${ }^{2}$, Jérôme Degert ${ }^{2}$, Marc Tondusson $^{2}$, Esben \\ SKovsen ${ }^{1}$, Eric Freysz ${ }^{2}$, and Emmanuel Abraham ${ }^{2}{ }^{*}$ \\ ${ }^{1}$ Department of Materials and Production, Aalborg University, Denmark \\ ${ }^{2}$ Univ. Bordeaux, CNRS, LOMA, UMR 5798, F-33400 Talence, France \\ *Corresponding author: emmanuel.abraham@u-bordeaux.fr
}

Compiled January 16, 2020

We demonstrate that the far-field terahertz beam generated from a Ti:Sapphire two-color laser-induced filament can exhibit a conical or Gaussian distribution depending on the filtering experimental conditions. Using both an incoherent Golay cell detector and a twodimensional coherent electro-optic detection covering the $0.2-3 \mathrm{THz}$ spectral range, in our experimental conditions, we provide evidences that the conical emission is due to photoinduced carriers in the silicon filter typically used to block the remaining pump laser light. Moreover, the terahertz beam retrieves an almost TEM $_{00}$ Gaussian spatial distribution when the silicon filter is preceded by a large bandgap ceramic filter, which stops the pump beam preventing carriers generation in the silicon filter. (c) 2020 Optical Society of America
OCIS codes: (110.6795) Terahertz imaging; (300.6495) Spectroscopy, terahertz;(350.5400) Plasmas; (140.3295) Laser beam characterization.

\section{http://dx.doi.org/10.1364/ao.XX.XXXXXX}

Two-color laser-induced air plasma filaments is a very convenient mean to generate intense ultrashort terahertz $(\mathrm{THz})$ pulses [1-4]. This THz emission has been described by different models such as two-dimensional (2D) transverse photocurrent [5], four-wave mixing [6], or a combination of the two as well. In short, within the plasma filament induced by a focused femtosecond millijoule optical pulse at a fundamental frequency $\omega$ and its second harmonic $2 \omega$, electrons are accelerated in a step-like response with a frequency content much higher than the one subtended by the envelope of the femtosecond two-color electric laser field [7].

The method presents obvious advantages such as relative simplicity, self-repairing gas media and possibility of high excitation laser intensity. These benefits enable unique applications for broadband $\mathrm{THz}$ time-domain linear spectroscopy. Non-linear spectroscopy requires tight focusing and, consequently, a full spatial characterization of $\mathrm{THz}$ radiation emitted from the air filament is necessary. In 2006, using a scanning aperture and an electro-optic coherent detection, Zhong et al. first reported a $\mathrm{THz}$ beam profile with an interference structure caused by the phase walk-off between the $\mathrm{THz}$ and the optical radiation propagating inside the filament [3]. This off-axis phase-matched so-called "conical" THz emission has also been observed using incoherent detections [8-10]. To account for this conical THz emission and considering the relative phase change between the two-color pulses along the plasma filament and the electron screening due to plasma density, various theoretical models have been proposed in 2006 [3], 2012 [8, 11] and 2014 [12]. A general consensus among the models is that higher frequencies are distributed in lower aperture angle cones [8, 11, 12]. In 2018, Ushakov et al. studied the $\mathrm{THz}$ beam profile as a function of the pump laser focusing conditions. They found that only low numerical apertures $(>10 \mathrm{~mm}$ long filaments, NA $<0.02)$ support conical emission [13]. This is contrary to the measurements in ref. [3], where a focusing lens with a $50 \mathrm{~mm}$ focal length showed a conical emission, whereas a $500 \mathrm{~mm}$ focal length exhibited a clear uni-modal one. Due to the lack of consensus on the emission profile characteristics in the community, more careful work is required.

Various experimental apparatus have been used to study the spatial transverse distribution of $\mathrm{THz}$ radiation from two-color air plasmas. Most works have been performed with detectors such as pyroelectric sensors or Golay cells [8-12]. The main disadvantage of these incoherent thermal detectors is their ultrabroadband spectral response. Even with the use of additional frequency filters such as silicon or high-density polyethylene (HDPE), it is always a challenge to limit the spectral response to the 0.1-5 THz region of interest. Very few papers were focused on characterizing the $\mathrm{THz}$ emission by using electro-optic sampling $[3,13]$. In all cases, point-by-point measurement or line scanning was used to reconstruct the spectrally-resolved 2D transverse $\mathrm{THz}$ beam distribution.

Another important experimental aspect in any two-color air plasma setup concerns the necessity to block the remaining powerful forward-propagating laser light emerging from the plasma filament, whose fluence can exceed $1 \mathrm{~mJ} / \mathrm{cm}^{2}$. Most experiments performed by Ti:Sapphire lasers use a semiconductor silicon wafer owing to its high resistance and low $\mathrm{THz}$ absorption. However, as previously noted in a few papers [10, 12], the energy of the photon of the laser pump light is significantly above the $1.24 \mathrm{eV}$ bandgap of silicon, meaning that the laser pulse can easily excite photocarriers in the silicon wafer. This induces a large increase of the silicon reflectivity near the $\mathrm{THz}$ beam center, 
decaying with the nanosecond lifetime of the excited carriers. The role of photoinduced losses in the silicon wafer has been studied using a Golay cell placed on a 2D raster-scan stage [10]. For long distances between the plasma and the silicon wafer (> $200 \mathrm{~mm}$ ), the conical $\mathrm{THz}$ emission exhibits an additional central peak. The authors concluded that this on-axis emission feature suggests a second emission mechanism in the plasma.

In this letter, similarly to ref. [13], we investigate the frequency-resolved angular distribution of the $\mathrm{THz}$ emission, in the $0.2-3 \mathrm{THz}$ range, generated by a Ti:Sapphire two-color laser-induced air plasma filaments. For that purpose, we used a real-time 2D electro-optic coherent detection scheme providing a direct characterization of the transverse $\mathrm{THz}$ electric field distribution. Similar to most previous works, the THz diverging emission has been measured just after the plasma to avoid any perturbations from additional optics such off-axis parabolic mirrors or lenses. We paid a special attention to the filter used to block the forward-propagating laser light after the plasma filament. We will discuss the influence of this filter on the $\mathrm{THz}$ beam propagation by comparing a small bandgap silicon filter with a ceramic filter. This latter presents a large bandgap $(>6 \mathrm{eV})$, high ablation resistance and decent $\mathrm{THz}$ transmission (60\% at $1 \mathrm{THz}, 1 \mathrm{~mm}$ filter thickness) [14].

The experiment is outlined in Fig. 1. An infrared laser pulse (50 fs, $800 \mathrm{~nm}, 2 \mathrm{~mJ}, R=5 \mathrm{~mm}$ beam waist, $1 \mathrm{kHz}$ repetition rate) is focused in air by a plano-convex lens L1 with a focal length $f$ ranging from $150 \mathrm{~mm}$ to $430 \mathrm{~mm}$. After passing through a BBO crystal for second harmonic generation, the resulting intense two-color laser pulse can produce a filament in air whose length depends on the numerical aperture NA defined as $\mathrm{NA}=\sin (\arctan (R / f))$. Then, an intense THz pulse is emitted from the plasma filament. Two different detection schemes have been used to characterize the $\mathrm{THz}$ emission. First, we used an incoherent detection composed of a Golay cell (GC) (Tydex GC-1P) connected to a lock-in amplifier, an entrance aperture (AP) with $3 \mathrm{~mm}$ diameter positioned at $150 \mathrm{~mm}$ from the plasma center and a combination of silicon (SF) and ceramic (CF) filters (Fig. 1). The silicon wafer (high resistivity float zone silicon, thickness $1 \mathrm{~mm})$ and the ceramic filter $\left(96 \% \mathrm{Al}_{2} \mathrm{O}_{3}\right.$, thickness $1 \mathrm{~mm}$ ) were provided by Tydex and CSC Ltd, respectively. Two experimental configurations were considered: while the position of the silicon filter is fixed, the ceramic filter can be either $50 \mathrm{~mm}$ before $(\mathrm{CF}(\mathrm{A}))$ or $5 \mathrm{~mm}$ behind $(\mathrm{CF}(\mathrm{B}))$ the latter. For both filtering configurations, the filters and the Golay cell are placed on a rotation stage in order to measure the amplitude of the $\mathrm{THz}$ transverse emission profile as a function of the half-opening angle (HOA), as defined in Fig. 1.

The second detection system consists in 2D electro-optic sampling (2DEOS) to measure the time-dependent spatial distribution of the THz electric field [15]. The THz beam is sent into a large aperture (20 mm diameter), $1 \mathrm{~mm}$ thick, $\langle 110\rangle \mathrm{ZnTe}$ crystal (Fig. 2), positioned $60 \mathrm{~mm}$ behind the center of the plasma filament. By 2DEOS with a time-delayed femtosecond laser probe pulse, we are able to transfer the $\mathrm{THz}$ electric field distribution onto the spatial profile of the laser probe beam, which is detected by a $256 \times 256$ pixels CMOS camera. For a given time delay between the $\mathrm{THz}$ and the probe pulses, the system is able to provide a 2D image at $800 \mathrm{~nm}$, corresponding to the distribution of the $\mathrm{THz}$ electric field. This image can be obtained with a $500 \mathrm{~Hz}$ acquisition rate thanks to the synchronization with the laser repetition rate and a dynamic subtraction method [16]. To analyze the data, each pixel was Fourier-transformed along its time delay-axis to provide the frequency-resolved $2 \mathrm{D} \mathrm{THz} \mathrm{am-}$

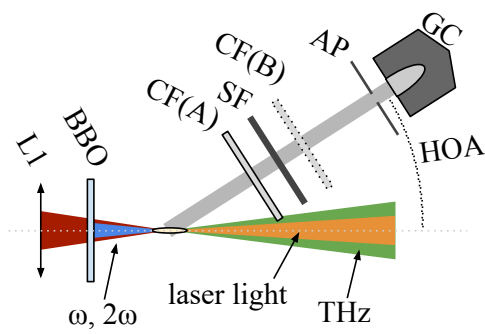

Fig. 1. Incoherent $1 \mathrm{D} \mathrm{THz}$ detection with a Golay cell. L1: plano-convex lens with focal length $f$. CF: ceramic filter, SF: silicon filter, AP: aperture, GC: Golay cell. HOA: half-opening angle. Two configurations were relevant: alumina first $\mathrm{CF}(\mathrm{A})$ or silicon first $\mathrm{CF}(\mathrm{B})$.

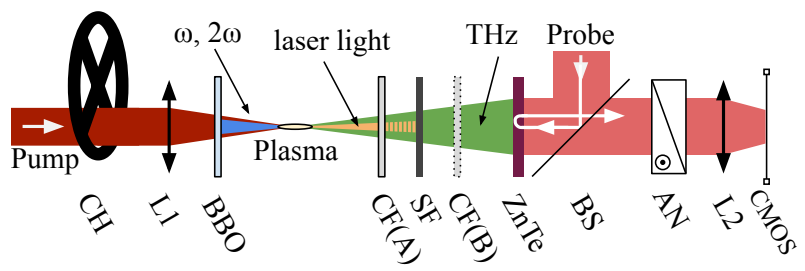

Fig. 2. Coherent 2D electro-optic sampling setup. CH: optical chopper for dynamic subtraction, L1: plano-convex lens with focal length $f$. CF: ceramic filter, SF: silicon filter, BS: beamsplitter, AN: analyzer, L2: objective lens $f=50 \mathrm{~mm}$. Two configurations were relevant: alumina first $(\mathrm{CF}(\mathrm{A}))$ or silicon first $(\mathrm{CF}(\mathrm{B}))$.

plitude and phase distribution of the THz electric field from 0.2 to $3 \mathrm{THz}$, limited by the spectral bandwidth of the ZnTe crystal and the filters. It is important to note that all data were corrected to account for the Gaussian transverse profile of the large probe laser beam (10 $\mathrm{mm}$ beam radius, measured at $\left.1 / e^{2}\right)$, which automatically decreases the $\mathrm{THz}$ amplitude out of the center of the beam. Again, two experimental configurations were considered. The silicon filter is placed $50 \mathrm{~mm}$ after the center of the plasma filament whereas the ceramic filter can be either $10 \mathrm{~mm}$ before $(\mathrm{CF}(\mathrm{A}))$ or $5 \mathrm{~mm}$ behind $(\mathrm{CF}(\mathrm{B}))$ the silicon one.

For a $f=300 \mathrm{~mm}$ focusing lens L1 (NA $=0.0167,5 \mathrm{~mm}$ filament length), Fig. 3 represents the incoherent $\mathrm{THz}$ emission profile as a function of the HOA. In the silicon first configuration $\mathrm{CF}(\mathrm{B})$, with a $2 \mathrm{~mJ}$ pump laser light illuminating the silicon wafer, we estimated that the laser light fluence on the silicon wafer is

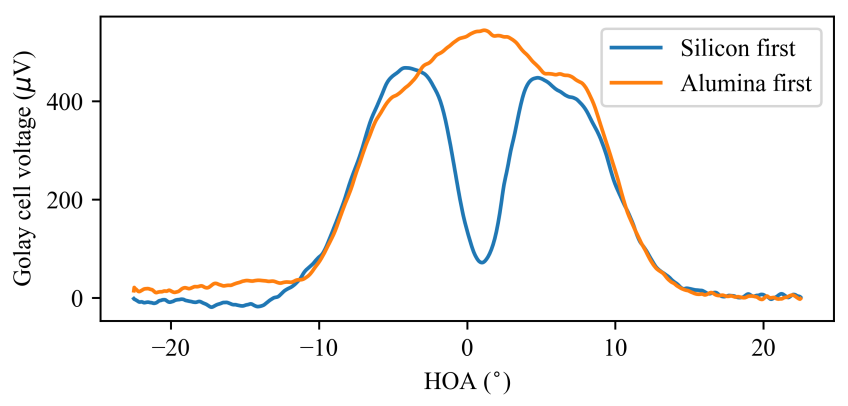

Fig. 3. Incoherent detection of the $\mathrm{THz}$ emission profile for the two experimental configurations: alumina first $\mathrm{CF}(\mathrm{A})$ and silicon first $\mathrm{CF}(\mathrm{B})$. 


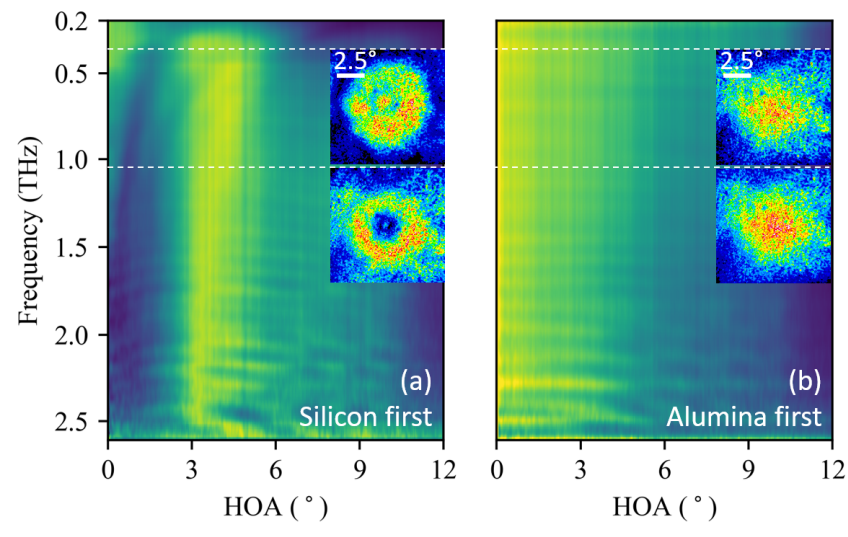

Fig. 4. 2DEOS evolution of $\mathrm{THz}$ amplitude as a function of frequency and HOA, in the case of silicon first $\mathrm{CF}(\mathrm{B})$ (left) and alumina first $\mathrm{CF}(\mathrm{A})$ (right). Yellow color indicates higher $\mathrm{THz}$ amplitude. Insets: 2D spatial distributions of the THz electric field at $0.37 \mathrm{THz}$ and $1.05 \mathrm{THz}$.

as high as $4 \mathrm{~mJ} / \mathrm{cm}^{2}$. The data plot shows a distinct depression 209 in the central part of the emission. The resulting conical THz 210 emission has a $\mathrm{HOA}$ of $5^{\circ}$ to $6^{\circ}$, in agreement with previous 211 results $[8,12]$. We can also notice that the hole diameter is in 212 the same range as the size of the laser pump light illuminating ${ }_{213}$ the silicon wafer. The situation is different for the alumina first ${ }^{214}$ case $\mathrm{CF}(\mathrm{A})$ where the $\mathrm{THz}$ emission is maximum in the center 215 like a Gaussian beam. We conclude that if the silicon filter is the 216 first optical element after the plasma filament, i.e. it is shined 217 by both the $\mathrm{THz}$ beam and the remaining laser pump light, the ${ }_{218}$ transmitted $\mathrm{THz}$ beam exhibits a conical emission. A similar 219 result has been obtained using a Si wafer from another company. 220 It is worth noting that, outside the center of the $\mathrm{THz}$ beam, both 221 configurations exhibit similar HOA.

As explained previously, the main disadvantage of the in- 223 coherent thermal detection obtained with the Golay cell is the 224 incapacity to completely block the high $\mathrm{THz}$ frequencies ranging 225 from 10 to $25 \mathrm{THz}$, even with the use of silicon, alumina and 226 HDPE filters. Therefore, we repeated the same measurement 227 with the 2DEOS imaging system, which also allows the direct 228 time-dependent measurement of the 2D THz electric field. Fig- 229 ure 4 represents the evolution of $\mathrm{THz}$ amplitude as a function of frequency and $\mathrm{HOA}$, in the case of silicon first $\mathrm{CF}(\mathrm{B})$ (left) and alumina first $\mathrm{CF}(\mathrm{A})$ (right). Here, $\mathrm{HOA}=\arctan (r / 60)$, where $r$ is the distance in mm between the center of the $\mathrm{THz}$ beam and the measured spot. The measurements have been performed with the $f=300 \mathrm{~mm}$ focusing lens L1 (NA $=0.0167$ ). For a given distance from the center of the beam, we averaged the $\mathrm{THz}$ amplitude along the azimuthal angle in order to get the 2D map presented in Fig. 4.

With the silicon filter first $\mathrm{CF}(\mathrm{B})$, the central beam hole is evident for frequencies higher than $0.5 \mathrm{THz}$ (Fig. 4, left). The HOA of this central dark region is about $3.5^{\circ}$ at $0.5 \mathrm{THz}$, slightly decreasing below $3.0^{\circ}$ at $2.5 \mathrm{THz}$. The two insets also show the 2D spatial distributions of the $\mathrm{THz}$ electric field amplitude, for different frequencies. At $1.05 \mathrm{THz}$ (dotted white line in Fig. 4), the $\mathrm{THz}$ beam exhibits a clear conical profile. For frequencies below $0.5 \mathrm{THz}$ (dotted white line at $0.37 \mathrm{THz}$ ), a central peak can be observed in the center of the bright ring. We believe that the silicon filter acts as a reflective or absorbing material for $\mathrm{THz}$ radiation in this region due to the presence of photo-carriers induced by the remaining pump laser light thus leading to the diffraction-like pattern observed below $0.5 \mathrm{THz}$. Let us note that the central depression has been observed down to the detection sensitivity limit, corresponding to a laser pump pulse energy of $250 \mu \mathrm{J}$ (data not shown). However, this still corresponds to an incident laser light fluence of nearly $0.4 \mathrm{~mJ} / \mathrm{cm}^{2}$ on the silicon wafer, which is sufficient for photo-carriers excitation. We also consider that the uni-modal $\mathrm{THz}$ emission below $0.5 \mathrm{THz}$ cannot be attributed to the plasma absorption since it is not observed in the alumina first case, in contradiction with ref. [13].

For the alumina first case $\mathrm{CF}(\mathrm{A})$, as previously observed with the incoherent detection, for all frequencies, no central hole is observed in the THz emission (Fig. 4, right). The THz emission exhibits a Gaussian-like profile with a slight decreasing FWMH if the frequency increases. This result is also shown in the two insets representing the 2D spatial distributions of the $\mathrm{THz}$ electric field amplitude at $0.37 \mathrm{THz}$ and $1.05 \mathrm{THz}$. The insets highlight here the clear Gaussian-like THz profiles.

To test our assumption of photo-carriers excitation in silicon filter induced by the laser light, we conducted a complementary experiment. With the 2DEOS setup and with the alumina filter first, we have enough space to irradiate the silicon filter with an oblique incidence $800 \mathrm{~nm}$ laser pulse $(10 \mu \mathrm{J}, 5 \mathrm{~mm}$ beam waist giving a light fluence of $10 \mu \mathrm{J} / \mathrm{cm}^{2}$ ), with a time delay $\tau$ with respect to the $\mathrm{THz}$ pulse peak controlled by a delay line. This additional laser beam overlaps the center of the THz beam at the silicon filter position with a light fluence which is 400 times smaller than the experiments depicted in, Figs. 3 and 4 . For various time delays $\tau$ between the $\mathrm{THz}$ pulse and the oblique infrared pulse, Fig. 5 shows the evolution of the 2D THz electric field sampled at its maximum. The red color indicates a positive electric field and the blue color a negative one (the white color reflects the absence of $\mathrm{THz}$ signal), confirming the divergence of the $\mathrm{THz}$ beam emerging from the plasma filament. For $\tau \leq 0$, the oblique laser pulse interacts with the silicon filter after the arrival of the $\mathrm{THz}$ pulse and we observe a typical divergent $\mathrm{THz}$ electric field distribution with a maximum amplitude in the center. Then, for positive values of $\tau$, we clearly observe the time-dependent photo-excitation of the silicon wafer by the laser pulse, with a right to left curtain effect due to the oblique incidence between the $\mathrm{THz}$ and laser beams. As a result, for $\tau \geq 4.00 \mathrm{ps}$, we observe a white central disk indicating the absence of THz signal in this area. This confirm our assumption

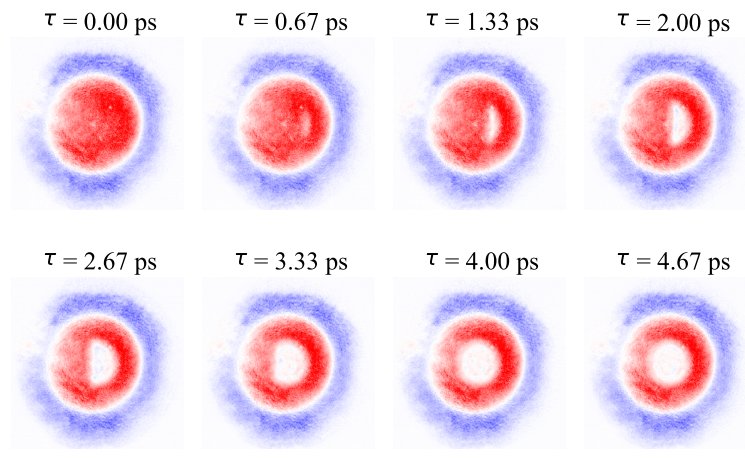

Fig. 5. Evolution of the 2D THz electric field as a function of time delays $\tau$ between the THz pulse and the additional oblique infrared laser pulse. Blue, white and red is for negative, zero and positive $\mathrm{THz}$ electric field, respectively. White ring angular diameter is $4.7^{\circ}$. The experimental configuration corresponds to alumina filter first $\mathrm{CF}(\mathrm{A})$. 


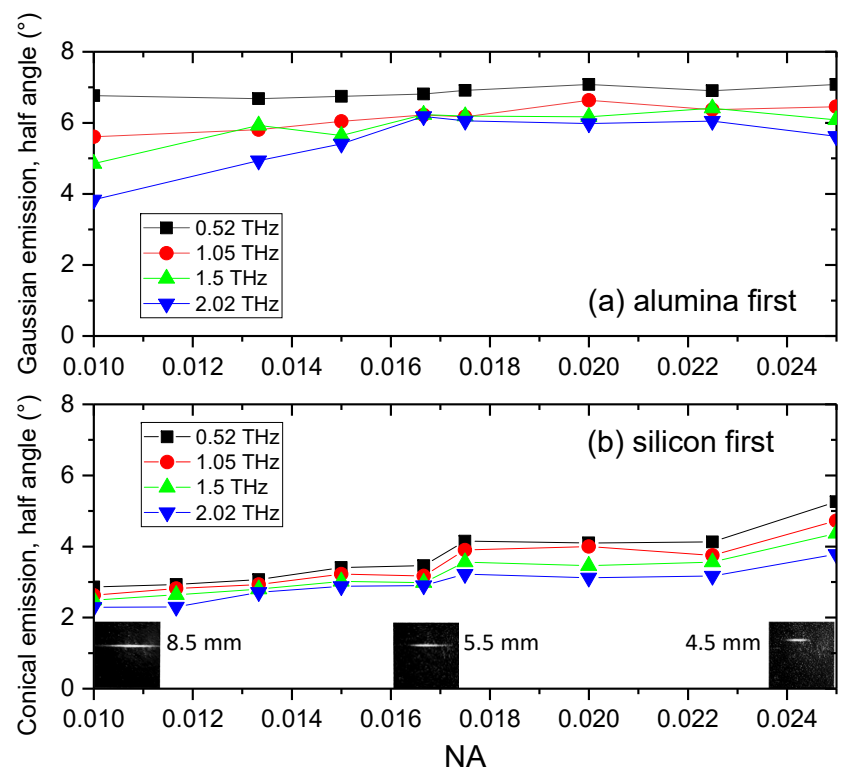

Fig. 6. (a) Alumina first. Evolution of the half angle of the Gaussian-like $\mathrm{THz}$ beam as a function of the numerical aperture NA. (b) Silicon first. Evolution of the half angle of the conical THz beam as a function of the numerical aperture NA. Insets: pictures of plasma filaments and filament lengths, image sizes are $10 \times 10 \mathrm{~mm}^{2}$.

of photo-carriers excitation in the silicon filter. The central $\mathrm{THz}$ beam is fully reflected (or absorbed) by the silicon filter and exhibit a conical distribution which can persist much longer than the laser pulse duration due to the nanosecond charge carrier lifetime in silicon.

Finally, we investigated the dependence of the far-field $\mathrm{THz}$ beam with respect to the laser focusing conditions. With the 2DEOS detection, we measured the frequency-resolved $\mathrm{THz}$ electric field as a function of the numerical aperture NA, from $\mathrm{NA}=0.01$ ( $f=500 \mathrm{~mm}, 8.5 \mathrm{~mm}$ plasma length) to $\mathrm{NA}=0.025$ ( $f=200 \mathrm{~mm}, 4.5 \mathrm{~mm}$ plasma length), as shown in Fig. 6. As 3 previously explained, data were corrected to take into account the Gaussian transverse profile of the probe laser beam. We first consider the case of alumina filter first (Fig. 6(a)). The data points have been obtained by fitting the $\mathrm{THz}$ beam profile with a Gaussian function. For a given NA, the THz half emission angle (corresponding to the half angular width of the beam at half maximum amplitude) decreases as the $\mathrm{THz}$ frequency increases, as previously observed in refs. [8, 11, 12]. For a given frequency, it is worth noting that the $\mathrm{THz}$ half emission angle barely increases (about $1^{\circ}$ ) as NA increases. It means that the $\mathrm{THz}$ far-field distribution, in the $0.2-3 \mathrm{THz}$ spectral range and 313 for this NA range, is not strongly governed by the laser focusing ${ }^{314}$ conditions. To our knowledge, this has never been observed ${ }^{3}$ previously. Uni-modal angular distribution had previously been observed in the $0.3-3 \mathrm{THz}$ range only for moderate focusing by Ushakov et al. (NA > 0.035) [13]. For long focal lengths as in Fig. 6(a) (NA < 0.02), only conical emission were reported so far. Especially, Ushakov et al. concluded that decreasing NA up to 0.02 is important to observe a ring-structure, which is in contradiction with our experiment. We can also notice that these authors observed a strong on-axis peak for NA = 0.02 , superimposed on the conical emission, which was not reproduced by their simulations. We then consider the case of silicon filter first (Fig. 6(b)). The graph shows the evolution of the half angle of the far-field conical $\mathrm{THz}$ beam as a function of NA. As previously observed, the conical angle increases if the frequency decreases or if NA increases $[8,11,12]$. This is consistent with the observation that if NA increases (tighter laser focusing), then the pump laser light can excite photo-carriers onto a larger area of the silicon wafer, resulting in a larger conical angle $\mathrm{THz}$ emission.

To conclude, we have shown that the silicon filter used to block the remaining laser light behind the Ti:Sapphire two-color air plasma filament has a strong influence on the existence of the far-field conical $\mathrm{THz}$ distribution. We concluded that this conical beam is due to photo-excited charge carriers in the silicon wafer. If the laser light was sufficiently scattered or absorbed by a preceding large bandgap alumina filter, we observed a TEM 00 Gaussian-like $\mathrm{THz}$ distribution from 0.2 to $3 \mathrm{THz}$, which was not strongly governed by the laser focusing conditions under our experimental conditions. These measurements call for further detailed simulations of the $\mathrm{THz}$ emission from a two-color plasma, which will require consideration of the full spatio-temporal wave propagation in the plasma.

Funding. The author acknowledges funding from the Danish Independent Research Fund grant (DFF-6111-00119, 2016) and the LAPHIA Student mobility grant 2019. The Conseil Régional Nouvelle Aquitaine and FEDER are also thanked for funding the equipments of the COLA platform at LOMA.

\section{REFERENCES}

1. D. J. Cook and R. M. Hochstrasser, Opt. Lett. 25, 1210 (2000).

2. M. Kress, T. Löffler, S. Eden, M. Thomson, and H. G. Roskos, Opt. Lett. 29, 1120 (2004).

3. H. Zhong, N. Karpowicz, and X.-C. Zhang, Appl. Phys. Lett. 88, 261103 (2006).

4. H. Roskos, M. Thomson, M. Kreß, and T. Löffler, Laser \& Photonics Rev. 1, 349 (2007).

5. T. Oh, y. s. You, and K. Kim, Opt. express 20, 19778 (2012).

6. J. Zhao, W. Liu, S. Li, D. Lu, Y. Zhang, Y. Peng, Y. Zhu, and S. Zhuang, Photon. Res. 6, 296 (2018).

7. K.-Y. Kim, J. H. Glownia, A. J. Taylor, and G. Rodriguez, IEEE J. Quantum Electron. 48, 797 (2012).

8. Y. S. You, T. I. Oh, and K. Y. Kim, Phys. Rev. Lett. 109 (2012).

9. P. Klarskov, A. C. Strikwerda, K. Iwaszczuk, and P. U. Jepsen, New J. Phys. 15, 075012 (2013).

10. V. Blank, M. D. Thomson, and H. G. Roskos, New J. Phys. 15, 075023 (2013).

11. A. V. Borodin, M. N. Esaulkov, I. I. Kuritsyn, I. A. Kotelnikov, and A. P. Shkurinov, JOSA B 29, 1911 (2012).

12. A. Gorodetsky, A. D. Koulouklidis, M. Massaouti, and S. Tzortzakis, Phys. Rev. A 89, 033838 (2014).

13. A. A. Ushakov, P. A. Chizhov, V. A. Andreeva, N. A. Panov, D. E. Shipilo, M. Matoba, N. Nemoto, N. Kanda, K. Konishi, V. V. Bukin, M. KuwataGonokami, O. G. Kosareva, S. V. Garnov, and A. B. Savel'ev, Opt. Express 26, 18202 (2018).

14. K. Z. Rajab, M. Naftaly, E. H. Linfield, J. C. Nino, D. Arenas, D. Tanner, R. Mittra, and M. Lanagan, J. Microelectron. Electron. Packag. 5, 2 (2008).

15. M. Brossard, H. Cahyadi, M. Perrin, J. Degert, E. Freysz, T. Yasui, and E. Abraham, IEEE Transactions on Terahertz Sci. Technol. 7, 741 (2017).

16. Z. Jiang, X. G. Xu, and X.-C. Zhang, Appl. Opt. 39, 2982 (2000). 


\section{FULL REFERENCES}

1. D. J. Cook and R. M. Hochstrasser, "Intense terahertz pulses by fourwave rectification in air," Opt. Lett. 25, 1210 (2000).

2. K.-Y. Kim, J. H. Glownia, A. J. Taylor, and G. Rodriguez, "High-Power Broadband Terahertz Generation via Two-Color Photoionization in Gases," IEEE J. Quantum Electron. 48, 797-805 (2012).

3. M. Thomson, V. Blank, and H. G. Roskos, "Terahertz white-light pulses from an air plasma photo-induced by incommensurate two-color optical fields," Opt. Express 18, 23173-23183 (2010).

4. H. Zhong, N. Karpowicz, and X.-C. Zhang, "Terahertz emission profile from laser-induced air plasma," Appl. Phys. Lett. 88, 261103 (2006).

5. P. Klarskov, A. C. Strikwerda, K. Iwaszczuk, and P. U. Jepsen, "Experimental three-dimensional beam profiling and modeling of a terahertz beam generated from a two-color air plasma," New J. Phys. 15, 075012 (2013).

6. V. Blank, M. D. Thomson, and H. G. Roskos, "Spatio-spectral characteristics of ultra-broadband $\mathrm{THz}$ emission from two-colour photoexcited gas plasmas and their impact for nonlinear spectroscopy," New J. Phys. 15, 075023 (2013).

7. Y. S. You, T. I. Oh, and K. Y. Kim, "Off-Axis Phase-Matched Terahertz Emission from Two-Color Laser-Induced Plasma Filaments," Phys. Rev. Lett. 109 (2012).

8. A. V. Borodin, M. N. Esaulkov, I. I. Kuritsyn, I. A. Kotelnikov, and A. P. Shkurinov, "On the role of photoionization in generation of terahertz radiation in the plasma of optical breakdown," JOSA B 29, 1911-1919 (2012).

9. A. Gorodetsky, A. D. Koulouklidis, M. Massaouti, and S. Tzortzakis, "Physics of the conical broadband terahertz emission from two-color laser-induced plasma filaments," Phys. Rev. A 89 (2014).

10. A. A. Ushakov, P. A. Chizhov, V. A. Andreeva, N. A. Panov, D. E. Shipilo, M. Matoba, N. Nemoto, N. Kanda, K. Konishi, V. V. Bukin, M. KuwataGonokami, O. G. Kosareva, S. V. Garnov, and A. B. Savel'ev, "Ring and unimodal angular-frequency distribution of $\mathrm{THz}$ emission from two-color femtosecond plasma spark," Opt. Express 26, 18202 (2018).

11. J. Jones, C. McEnnis, Y. Dikmelik, J. Spicer, D. Drewry, and M. LeahyHoppa, "Terahertz Time-Domain Spectroscopy of Aluminum Oxide for Thermal Protection Applications," in 48th AIAA/ASME/ASCE/AHS/ASC Structures, Structural Dynamics, and Materials Conference, (American Institute of Aeronautics and Astronautics, Honolulu, Hawaii, 2007).

12. K. Z. Rajab, M. Naftaly, E. H. Linfield, J. C. Nino, D. Arenas, D. Tanner, R. Mittra, and M. Lanagan, "Broadband Dielectric Characterization of Aluminum Oxide $\left(\mathrm{Al}_{2} \mathrm{O}_{3}\right)$," J. Microelectron. Electron. Packag. 5, 2-7 (2008).

13. M. Brossard, H. Cahyadi, M. Perrin, J. Degert, E. Freysz, T. Yasui, and E. Abraham, "Direct Wavefront Measurement of Terahertz Pulses Using Two-Dimensional Electro-Optic Imaging," IEEE Transactions on Terahertz Sci. Technol. 7, 741-746 (2017). 


\section{FULL REFERENCES}

1. D. J. Cook and R. M. Hochstrasser, "Intense terahertz pulses by fourwave rectification in air," Opt. Lett. 25, 1210 (2000).

2. K.-Y. Kim, J. H. Glownia, A. J. Taylor, and G. Rodriguez, "High-Power Broadband Terahertz Generation via Two-Color Photoionization in Gases," IEEE J. Quantum Electron. 48, 797-805 (2012).

3. M. Thomson, V. Blank, and H. G. Roskos, "Terahertz white-light pulses from an air plasma photo-induced by incommensurate two-color optical fields," Opt. Express 18, 23173-23183 (2010).

4. H. Zhong, N. Karpowicz, and X.-C. Zhang, "Terahertz emission profile from laser-induced air plasma," Appl. Phys. Lett. 88, 261103 (2006).

5. P. Klarskov, A. C. Strikwerda, K. Iwaszczuk, and P. U. Jepsen, "Experimental three-dimensional beam profiling and modeling of a terahertz beam generated from a two-color air plasma," New J. Phys. 15, 075012 (2013).

6. V. Blank, M. D. Thomson, and H. G. Roskos, "Spatio-spectral characteristics of ultra-broadband $\mathrm{THz}$ emission from two-colour photoexcited gas plasmas and their impact for nonlinear spectroscopy," New J. Phys. 15, 075023 (2013).

7. Y. S. You, T. I. Oh, and K. Y. Kim, "Off-Axis Phase-Matched Terahertz Emission from Two-Color Laser-Induced Plasma Filaments," Phys. Rev. Lett. 109 (2012).

8. A. V. Borodin, M. N. Esaulkov, I. I. Kuritsyn, I. A. Kotelnikov, and A. P. Shkurinov, "On the role of photoionization in generation of terahertz radiation in the plasma of optical breakdown," JOSA B 29, 1911-1919 (2012).

9. A. Gorodetsky, A. D. Koulouklidis, M. Massaouti, and S. Tzortzakis, "Physics of the conical broadband terahertz emission from two-color laser-induced plasma filaments," Phys. Rev. A 89 (2014).

10. A. A. Ushakov, P. A. Chizhov, V. A. Andreeva, N. A. Panov, D. E. Shipilo, M. Matoba, N. Nemoto, N. Kanda, K. Konishi, V. V. Bukin, M. KuwataGonokami, O. G. Kosareva, S. V. Garnov, and A. B. Savel'ev, "Ring and unimodal angular-frequency distribution of $\mathrm{THz}$ emission from two-color femtosecond plasma spark," Opt. Express 26, 18202 (2018).

11. J. Jones, C. McEnnis, Y. Dikmelik, J. Spicer, D. Drewry, and M. LeahyHoppa, "Terahertz Time-Domain Spectroscopy of Aluminum Oxide for Thermal Protection Applications," in 48th AIAA/ASME/ASCE/AHS/ASC Structures, Structural Dynamics, and Materials Conference, (American Institute of Aeronautics and Astronautics, Honolulu, Hawaii, 2007).

12. K. Z. Rajab, M. Naftaly, E. H. Linfield, J. C. Nino, D. Arenas, D. Tanner, R. Mittra, and M. Lanagan, "Broadband Dielectric Characterization of Aluminum Oxide $\left(\mathrm{Al}_{2} \mathrm{O}_{3}\right)$," J. Microelectron. Electron. Packag. 5, 2-7 (2008).

13. M. Brossard, H. Cahyadi, M. Perrin, J. Degert, E. Freysz, T. Yasui, and E. Abraham, "Direct Wavefront Measurement of Terahertz Pulses Using Two-Dimensional Electro-Optic Imaging," IEEE Transactions on Terahertz Sci. Technol. 7, 741-746 (2017). 